\title{
Large-Scale Structure and Direction of Rotation in Galaxies
}

\author{
G. DE Vaucouleurs \\ Lowell Observatory, Flagstaff, Arizona
}

\section{A. LARGE-SCALE STRUCTURE}

$T^{\mathrm{H}}$ HE large-scale distribution of gas and dust in external galaxies seems to be closely correlated with the spiral arms and other main structural patterns. This was shown, in particular, by Baade and Mayall at the 1949 Symposium. A great many structural patterns exist among external galaxies; it is likely that these will prove to be important indicators of the dynamics and possibly of the evolution of cosmic gas clouds in stellar systems. This was pointed out at the 1953 Symposium by Hubble, whose original classification system was described in the report by Blaauw which includes also some indications given by Sandage of the program of revision and illustration of this system.

A similar and initially independent program had been initiated by the author in 1952 at Mount Stromlo in connection with a survey of the brighter southern galaxies $^{1}$; this was extended to the northern galaxies during a stay at Mount Hamilton in 1955. Through the kindness of Dr. Allan Sandage, and by permission of Dr. Ira S. Bowen, an advance copy of the plates for the forthcoming Hubble Memorial Volume and some of Hubble's unpublished notes were supplied to the author in 1956 to supplement the material previously available for a systematic morphological study and the construction of a revised classification system of external galaxies. Details of the revised, three-dimensional

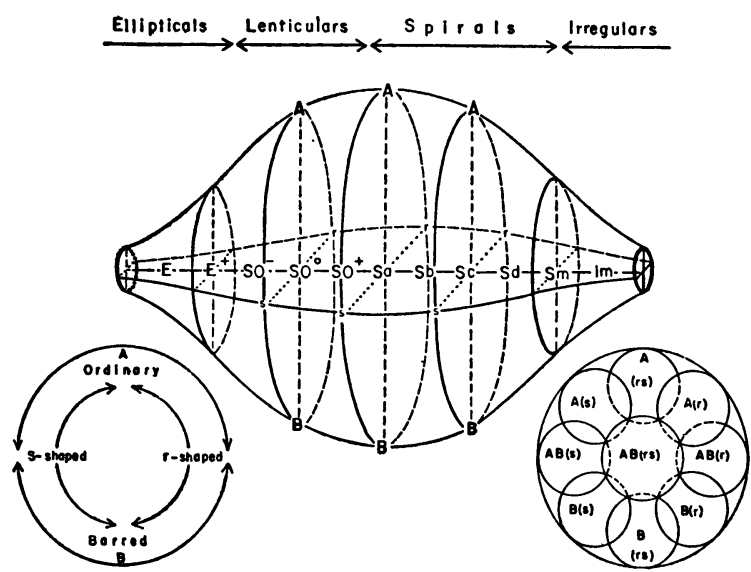

FIG. 1. Three-dimensional classification system of external galaxies, illustrating the notation system and the main stages in the four classes E, SO, S, I along the central sequence.

${ }^{1}$ G. de Vaucouleurs, Mem. Commonwealth Obs., Canberra, III, No. 13 (1956). classification will be published and fully illustrated elsewhere. ${ }^{2}$ The essential points are summarized below.

The three-dimensional classification appears necessary to represent objects of mixed characteristics in correct relation to the main sequences as well as the progressive divergence of the various sequences from $\mathrm{E}$ to $\mathrm{SO} / \mathrm{a}$ through the SO stages and their ulterior convergence from $\mathrm{SO} / \mathrm{a}$ to $\mathrm{I}$ through the $\mathrm{S}$ stages (Fig. 1). The present classification scheme and notation system rest on the following principles.

Four classes are retained: ellipticals E, lenticulars $\mathrm{SO}$, spirals S, irregulars I, which coincide with the main divisions introduced by Hubble.

The two families of lenticulars and spirals, originally denoted "normal" S and "barred" SB by Hubble, are now designated "ordinary" SA and "barred" SB, so as to permit the use of the compound symbol SAB for "intermediate" objects of mixed characteristics. The symbol $\mathrm{S}$ alone is used when a spiral object cannot be more accurately classified either SA or SB because of poor resolution, unfavorable tilt, etc. The "ordinary" spirals are not more "normal" than those of the "barred" family which are at least as common.

In the new class of lenticulars the two families are denoted SAO and SBO, depending on the absence or presence of a bar structure across the central lens; "intermediate" objects with a very weak bar are noted SABO. The symbol SO, used for SAO in Hubble's notation, is now used for a lenticular object which cannot be more precisely classified as either SAO or $\mathrm{SBO}$; this is often the case for edgewise objects.

Two main varieties are recognized in each of the lenticular and spiral families, the "annular" or "ringed" type, denoted (r), and the "spiral" or "S-shaped" type, denoted (s). Intermediate types are noted (rs). In the "ringed" variety the structure includes circular (sometimes elliptical) arcs or rings (SO) or consists of spiral arms or branches emerging tangentially from an inner circular ring (S). In the "spiral" variety two main arms start at right angles from a globular or little elongated nucleus (SA) or from an axial bar (SB).

The distinction between the two families $\mathrm{A}$ and $\mathrm{B}$ and between the two varieties ( $\mathrm{r}$ ) and (s) is most clearly marked at the transition stage $\mathrm{SO} / \mathrm{a}$ between the $\mathrm{SO}$ and $\mathrm{S}$ classes. It vanishes at the transition stage between $\mathrm{E}$ and $\mathrm{SO}$ on the one hand, and at the transition stage between $\mathrm{S}$ and $\mathrm{I}$ on the other.

${ }^{2}$ G. de Vaucouleurs, Handbuch der Physik (Springer Verlag, Göttingen, to be published), Vol. 53. 
Four subdivisions or stages are distinguished along each of the four spiral sequences $\mathrm{SA}(\mathrm{r}), \mathrm{SA}(\mathrm{s}), \mathrm{SB}(\mathrm{r})$, $\mathrm{SB}(\mathrm{s})$, viz., "early," "intermediate," and "late" denoted $a, b, c$ as in the standard classification, with the addition of a "very late" stage, denoted $d$. Intermediate stages are noted Sab, Sbc, Scd. The transition stage towards the Magellanic irregulars (whether barred or not) is noted Sm, e.g., the Large Magellanic Cloud is SB(s)m.

Along each of the nonspiral sequences the signs + and - are used to denote "early" and "late" subdivisions; thus $\mathrm{E}^{+}$denotes a "late" $\mathrm{E}$, the first stage of the transition towards the SO class. In both the SAO and $\mathrm{SBO}$ subclasses three stages, noted $\mathrm{SO}^{-}, \mathrm{SO}^{0}, \mathrm{SO}^{+}$are thus distinguished; the transition stage between $\mathrm{SO}$ and $\mathrm{Sa}$, noted $\mathrm{SO} / \mathrm{a}$ by Hubble, may also be noted $\mathrm{Sa}^{-}$. Notations such as $\mathrm{Sa}^{+}, \mathrm{Sb}^{-}$may be used occasionally in the spiral sequences, but the distinction is so slight between, say, $\mathrm{Sa}^{+}$and $\mathrm{Sb}^{-}$, that for statistical purposes it is convenient to group them together as Sab, etc. Experience shows that this makes the transition subdivisions, Sab, Sbc, etc., as wide as the main subdivisions, $\mathrm{Sa}, \mathrm{Sb}$, etc.

The classification of "irregulars" is still somewhat in doubt; objects obviously related to the Magellanic type, but which do not show clearly the characteristic spiral structure, are noted $I(m)$; those with an elongated core and asymmetrical branches are probably later stages of the SB sequences, e.g., NGC 4449, 6822, while those more nearly symmetrical and without bar-like core are probably later stages of the SA sequences, e.g., IC 1613, IC 2574; the distinction vanishes in the ultimate, chaotic, dwarf stages of low surface brightness, e.g., the Wolf-Lundmark Nebula or the Sextans System. ${ }^{3}$

A faint, outer, ring-like structure is often observed in lenticulars and early-type spirals which is not clearly related to a specific type; i.e., with some variants it appears about equally in all four sequences near the transition stage $\mathrm{SO} / \mathrm{a}$. This particularity which seems therefore more characteristic of a certain stage along the sequences than of any definite sequence, is denoted by an (R) preceding the symbol of the class. Examples of (R)Sa are NGC 1068 (M 77), NGC 4736 (M 94), NGC 7217, etc. of (R)SB: MGC 1291, 1326, 2859 , etc. Some objects have both an outer (R) structure and an inner (r) pattern; a good example is NGC 6753, noted $(\mathrm{R}) \mathrm{SA}(\mathrm{r}) \mathrm{ab}$.

In the spiral sequences supplementary data of some interest are the multiplicity of the spiral pattern and the character, "massive" or "filamentary" of the spiral arms. These are included whenever possible as subscripts, respectively, after the family and variety

\footnotetext{
${ }^{3}$ Objects of low surface brightness (when it is not obviously due to local obscuration, e.g., as for IC 10, IC 342) are almost invariably dwarf systems; this particularity may be noted by a (d) preceding the symbol of the class which is usually either $\mathrm{E}$ or $\mathrm{I}$. The existence of dwarf lenticulars and spirals remains to be demonstrated. Criteria are still lacking for the assignment of objects to the giant and supergiant groups on purely morphological grounds.
}

symbols. Thus M 33 is noted $\mathrm{SA}(\mathrm{s})_{2} \mathrm{C}_{m}$ and described as "an ordinary, late-type, S-shaped spiral with two main massive arms." When one or more additional and weaker arms are present this is noted $2+1$ (e.g., M 99), 2+2 (e.g., $M$ 51), etc. An asymmetrical, regular spiral with one arm stronger than the other as is often the case among late-type barred spirals (e.g., NGC 7479) is noted 1+1 (not 2). Branching arms not clearly assignable to a definite multiplicity are noted $1^{+}, 2^{+}$, etc., as the case may be; this is a frequent occurrence among late-type spirals. A complicated spiral pattern of multiplicity higher than 4 , as often observed in late-type spirals, is simply noted $n$ (e.g., NGC 2903, 7793); this, however, occurs also among early-type spirals of the ringed variety (e.g., NGC 4736, 7217).

Edge-wise systems which can usually be classified only as E7, SO, S(a,b,c,d), or I are noted (sp) for "spindle." For ellipticals the traditional notation E0, E1, ‥ E7 may be preserved, although it is not homogeneous with the rest of the classification scheme and notation system used for the other classes; it is really almost superfluous when measured dimensions and flattening are given.

Figure 2 illustrates the main criteria used to distinguish between families and varieties in a central cross section of the classification volume near the stage $\mathrm{Sb}$. The classification criteria are described in relation to the three main parts of a galaxy defined by Hubble.

(a) The nucleus, i.e., the very small, very bright, central condensation often sharply defined as the center of symmetry of the structure; it is round in SA, elliptical in SB.

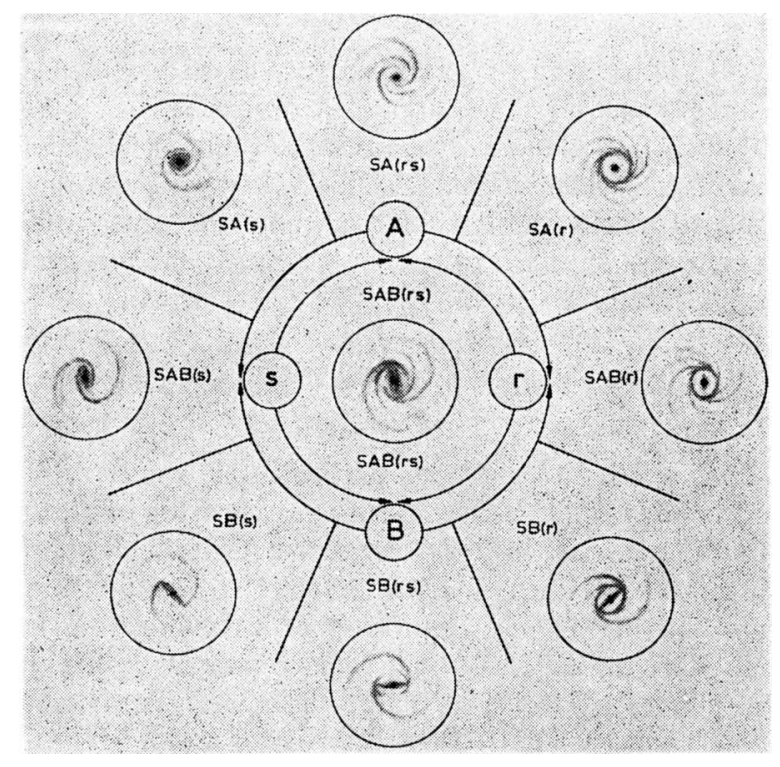

FIg. 2. Cross section of the classification volume near the stage $\mathrm{Sb}$, illustrating the main types of spiral galaxies. Above: ordinary family SA; below: barred family SB. Left: S-shaped variety $\mathrm{S}(\mathrm{s})$; right: ringed-shaped variety $\mathrm{S}(\mathrm{r})$. Note transition types $\mathrm{SAB}, \mathrm{S}(\mathrm{rs})$, and mixed types in center. 
(b) The lens, smooth, bright, and sharply defined in the lenticulars and early spirals, being crossed by the bar in SB and brighter at the edge in S(r).

(c) The envelope, generally faint and smooth with indefinite outer boundary in the lenticulars, brighter and occupied by the spiral arms in spirals. The outer (R) structure appears often in its outer parts near the stage $\mathrm{SO} / \mathrm{a}$.

The distinction between these three regions is most definite near the middle of the classification sequence (SO/a) and vanishes at $\mathrm{E}$ and $\mathrm{Im}$.

The spirals of the ordinary family SA are above, of the barred family SB below; the ringed variety $S(r)$ is to the right, the S-shaped variety $S(s)$ to the left. The mixed types $\mathrm{SAB}(\mathrm{rs})$ are in the center. There is a continuous transition between each type and only the main forms are illustrated; actual objects can occupy any intermediate position in the plane (volume).

The main classification criteria follow.

\section{SA(r)}

$\mathrm{SA}(\mathrm{r})$ has a small, sharp, very bright, and round nucleus isolated in the center of a circular ring at the edge of which emerge many tightly wound filamentary spiral arms or arcs; the nucleus and ring often merge and disappear in the over-exposed image of the central bulge, but the high multiplicity of the spiral pattern is usually sufficient for identification; a weak outer ring (R) made up of many closely coiled spiral arcs is often present in early stages of this sequence. Examples are NGC 488, 6753, 7217; other examples: NGC 4736 (M 94), Sab and 5055 (M 63), Sbc.

\section{SA(rs)}

SA(rs) has a fairly small, fairly sharp, bright, and round nucleus in the center of a diffuse lens or bulge out of which two main arms and two or more additional, weaker arms emerge tangentially; the two main arms simulate an incomplete ring around the lens; weak outer arms simulate an (R) structure in the early stages. Examples are NGC 1068 (M 77), 3147, 4237, possibly NGC $3521,4800,7079,7590$.

\section{SA(s)}

This is the typical, regular logarithmic spiral; it has a fairly large, diffuse, round nucleus extending smoothly into the circular or little elongated lens which shows some spiral pattern of dark matter; two main spiral arms with occasional branching or weaker secondary arms start at the rim of the lens. Examples are NGC 3031 (M 81), Sab, 4569, 7205, 7331. One diameter of the lens is occasionally slightly brighter and longer and the outer arms may have a tendency to "return" to the lens, indicating a transition toward the $\mathrm{SAB}(\mathrm{s})$ type; examples are NGC 224 (M 31), Sb and 4321 (M 100), Sbc.

\section{$\mathbf{S A B}(\mathbf{s})$}

$\mathrm{SAB}(\mathrm{s})$ has a fairly small, bright, elongated nucleus crossed by a weak, twisted dark lane in the center of a weak and broad bar marking the major axis of a faint, diffuse, and elongated lens with much spiral pattern of dark matter having a tendency to run parallel to the bar. Two main arms emerge near the extremities of the bar along a smooth, curved path, turning sharply just outside the lens; their faint extremities tend to return to the lens along an almost circular outer loop, simulating an (R) structure in the early stages. Examples are NGC 1566, NGC 5236 (M 83), Sbc, 4579, Sab and 7392.

\section{SB $(\mathbf{s})$}

This is the typical "S-shaped" barred spiral; it has a small, very bright, and elongated nucleus distorted by a strong, twisted, dark lane as it crosses over from one side to the other of the strong, narrow bar marking the major axis of a much elongated lens. Two strong, main arms start sharply at right angles to the bar at both ends and return faintly to it after completing a turn of a quasi-circular loop. Examples are NGC 1097, $1300,1365,5383$. Later stages in this sequence are markedly asymmetrical as NGC 7479, Sc and 7741, Scd. Some, like NGC 1300, have a third, fainter arm forming a half-ellipse close to the lens and indicating a transition toward the SB(rs) type.

\section{SB $(\mathbf{r s})$}

$\mathrm{SB}(\mathrm{rs})$ has a fairly small, very bright and elongated nucleus crossed by a twisted dark lane, as in $\mathrm{SB}(\mathrm{s})$, in a very strong, very narrow bar marking the major axis of an elongated lens whose rim is brighter, especially near the extremities of the bar; this produces a characteristic "dash-dot in parentheses" pattern, thus (-.-), from which emerge two main spiral arms with faint additional branches near the rim of the lens. Examples are NGC 4548, 4593, 7124. Some, like NGC 4349, Sab, having a brighter lens and more continuous rim mark the transition toward $\mathrm{SB}(\mathrm{r})$.

\section{SB $(\mathbf{r})$}

This is the typical " $\Phi$-shaped" barred spiral. It has a fairly large, elongated nucleus with weak, spiral, dark lane in a strong, narrow bar along the major axis of an elliptical ring marking the edge of the lens. Two main arms start tangentially from the ring near the extremities of the bar (i.e., at right angles to the bar); one or two fainter arms branch out from breaks in the ring near its minor axis. In early stages the main arms tend to form an outer, circular (R) structure, as in NGC 1433; in later types the breaks in the ring tend to produce slightly hexagonal shapes. Examples are NGC 1433, Sa or Sab, 3185, 3351, Sab, 2523, Sbc. 


\section{$\mathrm{SAB}(\mathbf{r})$}

$\mathrm{SAB}(\mathrm{r})$ has a fairly small, little elongated nucleus in a fairly broad and faint bar marking the major axis of a little elongated ring from which several spiral arms branch out. The main arms have a slight tendency to "return" inward. Examples are NGC 1832, 7531, Sab, 6744, Sbc. Some like NGC 6902, 6935 (Sa), 6937 , have only very faint traces of a bar and mark the transition toward $\mathrm{SA}(\mathrm{r})$.

\section{$\mathrm{SAB}(\mathbf{r s})$}

This is the most general mixed type involving all possible transitions between the main typical patterns. An almost infinite variety is possible here, but for classification purposes the main characteristics of this hybrid type are a small bright nucleus in a broad diffuse bar with some spiral structure in the lens. The bar crosses a nearly circular or of ten hexagonal pseudoring formed by the inner sections of the spiral arms. A very good example is NGC 4304 (M 61), Sbc; other examples are NGC 3145,6814 . At a later stage this mixed structure is well illustrated by the central regions of NGC 5457 (M 101) and NGC 6946, both Scd.

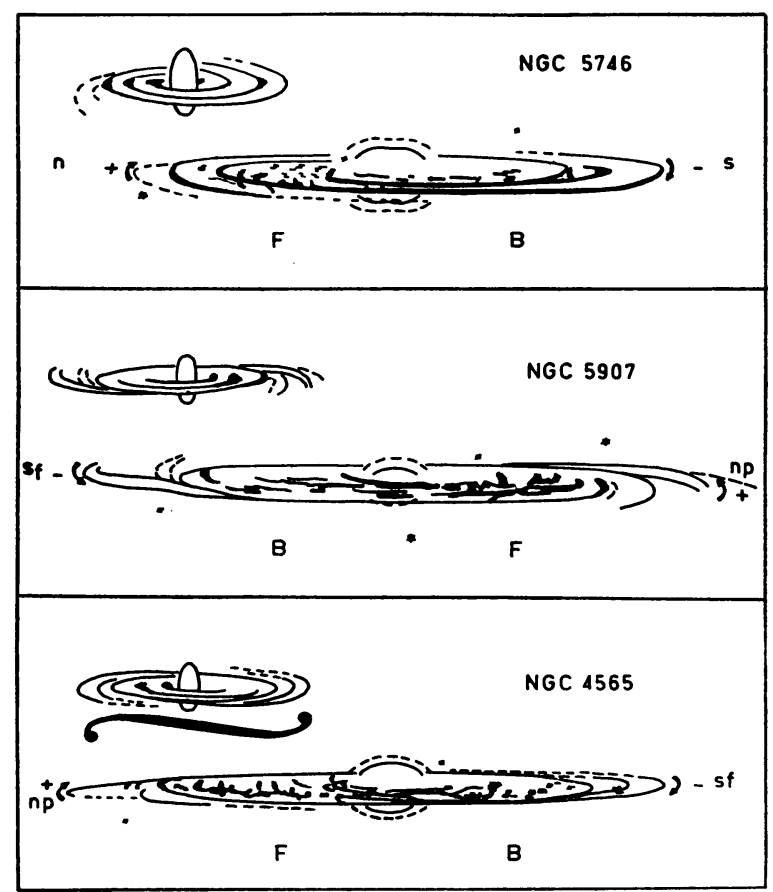

FIG. 3. Determination of the sense of the spiral pattern in nearly edgewise spirals. The sense of the spiral pattern is directly traceable in NGC 5746; it is indicated by the faint extremities of the arms in NGC 5907 and by the related "twist" of the outline of the disk in NGC 4565. The sense of the spiral pattern is also related to the longitudinal asymmetry of the luminosity distribution as indicated by the letters B (bright) and F (faint) in each case. The orientation and the direction of the spectroscopic rotation are marked. All sketches are traced from 200 -inch photographs.
Revised types in this classification system have been determined for over 1500 bright galaxies of both hemispheres; they will be published together with revised magnitudes, dimensions, etc. in a forthcoming reference catalog of bright galaxies based on the classical Harvard Survey. It appears that about $90 \%$ of the brighter galaxies can be fitted into this scheme; the remaining $10 \%$ include badly distorted, interacting, and colliding systems such as NGC 1275, 1487, 4038-9, 3256 , and peculiar objects with unusual characteristics such as the M 82 type, Haro's blue galaxies, Mayall's Nebula, etc., the precise classification of which remains a task for the future.

\section{B. TILT CRITERIA AND DIRECTION OF ROTATION}

We now review briefly the observational evidence on the direction of rotation of the spiral pattern in ordinary and barred spirals. ${ }^{4}$ Reasons for the divergences in the interpretation of various objects according to the classical (Slipher-Hubble) viewpoint and according to the Stockholm school were analyzed. A list of critical objects was assembled which permits an unequivocal solution of the ambiguities from a variety of criteria, both classical or new, applied to structural details

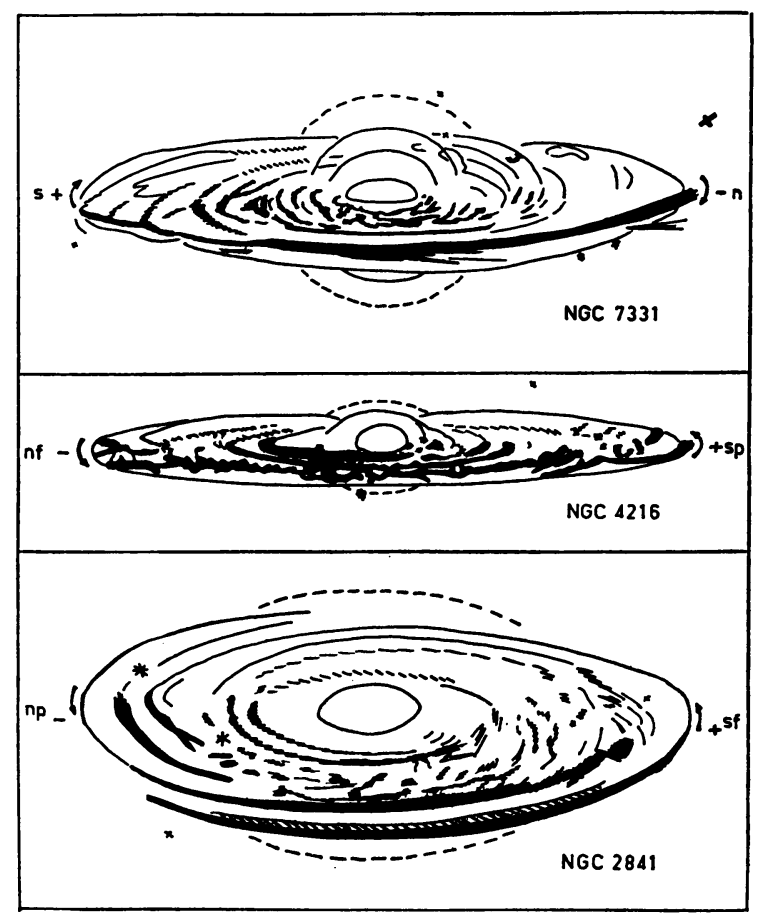

FIG. 4. Determination of the near side in tilted spirals. The near side of the disk is indicated in NGC 7331, NGC 4216 , and possibly NGC 2841 by the primary criterion of tilt, namely the projection of the outer dark lane against the outer regions of the central bulge traced from 200 -inch photographs. It is also indicated by the geometry of the bright arms and dark lanes near the apices of the projected disk as in Fig. 3.

'G. de Vaucouleurs, Astrophys. J. 127, 487 (1958). 


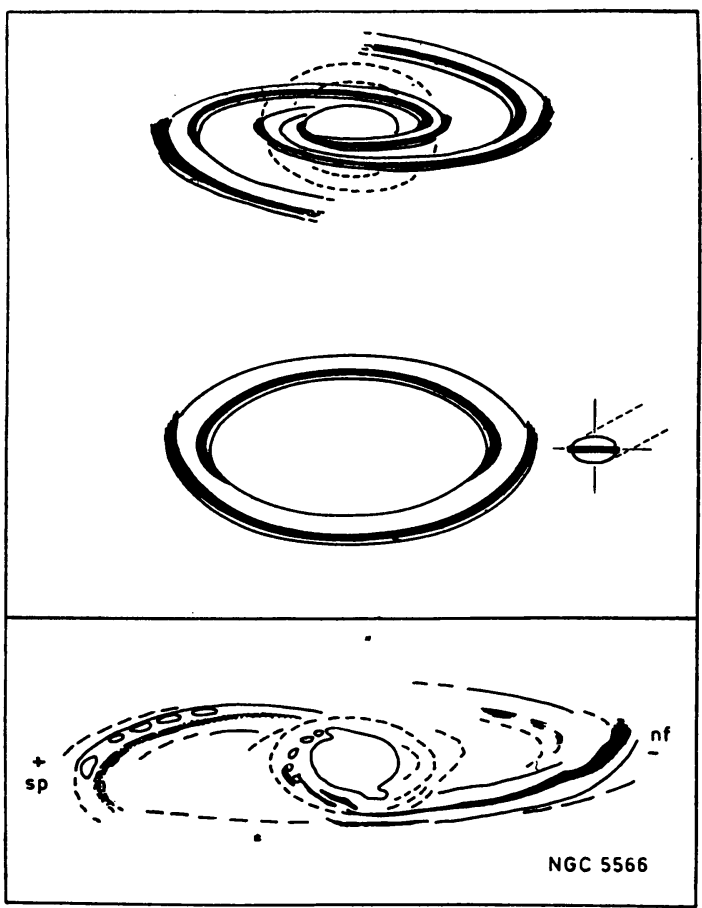

Fig. 5. Criterion of tilt based on the geometry of the bright arms and dark lanes. The relative path of the dark lane with respect to a bright toroid or spiral arm indicates whether it follows the concave or convex side of the arm. An application to the barred spiral NGC 5566 showing both inner and outer dark lanes identifies the near side and consequently the sense of rotation.

visible on the best $\mathrm{Mt}$. Wilson-Palomar photographs (made available by courtesy of Dr. I. S. Bowen) supplemented by new Lick spectra (kindly secured by Dr. N. U. Mayall).

For objects whose tilt angle between the equatorial plane and the line of sight is very small, such as NGC $891,4244,4565,4594,5746,5907$, there is general agreement that the primary absorption lane is on the near side. The 200-in. photographs indicate clearly the sense of the spiral pattern in NGC 4244, 4565, 5746 , and 5907; in all cases the arms are found to be "trailing" in the rotation (convex side preceding).

For objects whose tilt angle is somewhat larger, such as NGC 4216 and 7331, the sense of the spiral pattern is clear, but the interpretation of the obscuration pattern was in doubt. The 200-in. photographs show unmistakably, by repeated copying, the outer isophotes of the central bulge emerging on both sides of the minor axis "above" and "below" the spiral pattern; this established beyond question the location of the strong outer dark lanes on the near side of the disk and consequently the arms are trailing.

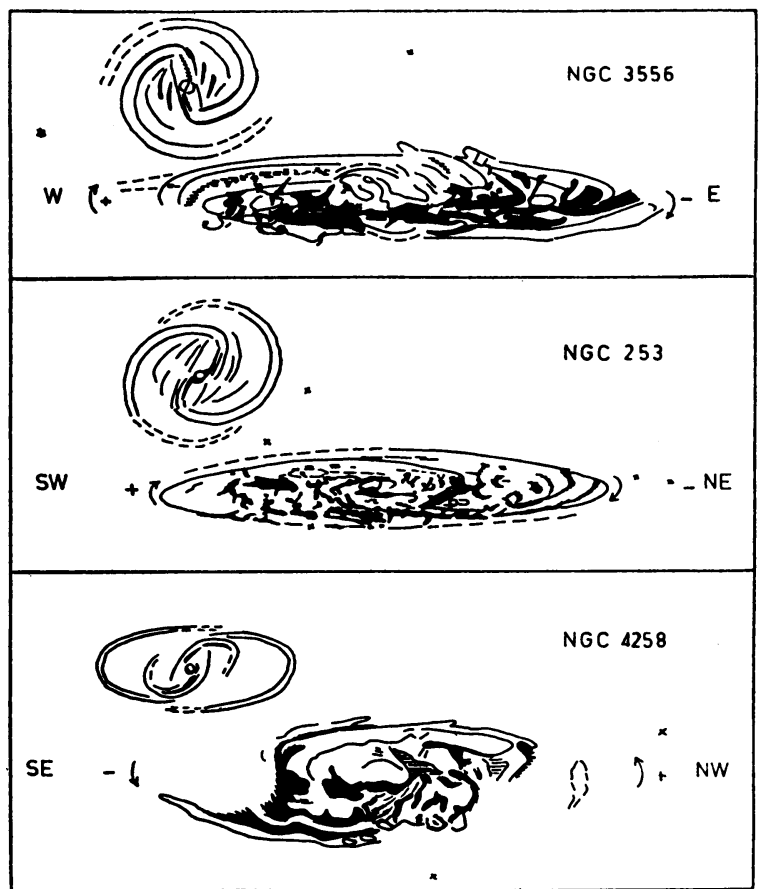

Fig. 6. Rotation and tilt of barred spirals. The sense of the spiral pattern in the edgewise system NGC 3556 is indicated by the outer parts of the arms; the near side is identified in NGC 253 by analogy with NGC 3556 and 5566 and in NGC 4258 by the obscuration pattern in its central lens which is similar to that in NGC 253. In all cases the arms are trailing in the rotation.

An additional tilt criterion based on the geometry of the relative locations and shapes of the lanes of dark matter with respect to the bright spiral arms makes it possible to identify the near side in galaxies showing both inner and outer dark lanes, such as NGC 2442, $3623,4216,5566$. The latter, type $\mathrm{SB}(\mathrm{rs}) \mathrm{a}$, is a new and especially fine example on 200-inch photographs. A recent Lick spectrogram indicates that the arms are trailing as in the other cases.

From the unequivocal cases solved by the "primary" geometric criteria, "secondary" photometric criteria are derived, based on the longitudinal asymmetry of the luminosity distribution along the major axis or the relative contrast of the dark lanes on either side of it. The former applies mainly to spirals of moderate or small tilt and indicates the sense of the spiral pattern even in cases where it is not directly recognizable; the latter applies mainly to spirals of moderate or large tilt and indicates the near side even when the primary absorption pattern is no longer visible. In all cases where the required spectroscopic data are available, these criteria also indicate that the arms are "trailing." Figures 3 to 6 illustrate these various points. 